\title{
Layer-By-Layer Design and Optimization of Xerogel-Based Amperometric First Generation Biosensors for Uric Acid
}

\author{
Grace E. Conway, ${ }^{\dagger}$ Raef H. Lambertson, ${ }^{\dagger}$ Margaret A. Schwarzmann, Michael J. Pannell, Helene \\ W. Kerins, Kristen J. Rubenstein, Jonathan D. Dattelbaum, and Michael C. Leopold* \\ Department of Chemistry, Gottwald Center for the Sciences, University of Richmond \\ Richmond, VA 23173
}

\begin{abstract}
A layer-by-layer (LbL) strategy of modifying an electrode with a specific combination of polymeric and xerogel materials is used to create an effective first generation biosensor for uric acid (UA) - a clinically relevant molecule implemented in pregnancy induced hypertension (PIH) diagnosis, a condition that can lead to a serious disorder called preeclampsia. In addition to offering a new, promising sensor for UA, this study represents significant progress for amperometric biosensor development since the strategy and materials employed were successfully and readily adapted from a glucose biosensor model scheme and used for the effective detection of UA, a fundamentally different molecule compared to glucose. Specifically, each of four, functional modifying layers (outer polyurethane (PU) selective membrane, the inner selective electropolymer, and the xerogel bi-layer) are systematically investigated and tailored for UA permeability and interferent discrimination. The role of PU hydrophobicity and its UA permeability are established while the enzyme-doped and outer diffusional xerogel layers are evaluated for uricase (UOx) species/loading and silane precursor dependence, respectively. LbL systematic evaluation reveals the specific combination of hydroxylmethyl triethoxy silane (HMTES) xerogels, a polyluminol-aniline electropolymer, and $100 \%$ hydrophilic polyurethane yields impressive uric acid sensing performance: effective sensitivity $(0.8 \mathrm{nA} / \mu \mathrm{M})$, linear response across physiologically relevant UA concentrations $(100-700 \mu \mathrm{M})$, fast response times $(\sim 10 \mathrm{sec})$, low limits of detection $(<$ $10 \mu \mathrm{M})$, and selectivity against most common interferents. Towards the specific application of PIH risk assessment, the optimized sensor exhibited 10 day stability as well as effective shelf-life exceeding 35 days. The presented system rivals or exceeds UA biosensor performance found in the literature and offers the possibility of miniaturization for in situ or in vivo remote diagnostic sensing. The successful adaptation suggests that the strategy and materials may be applicable to detecting/monitoring other medically significant molecules via sensor development.
\end{abstract}

${ }^{\dagger}$ These authors contributed equally to this work.

* To whom correspondence should be addressed. Email: mleopold@ richmond.edu. Phone: (804) 287-6329. Fax: (804) 287-1897

Keywords: electrochemical biosensor, amperometric, uric acid, xerogel, layer-by-layer, pregnancy-induced hypertension 


\section{Introduction}

In the biosensor technology field, it is relatively common that individual literature reports focus on systems, strategies, and materials that are selected, designed, and developed for the detection of a very specific target analyte. Over the past two decades, many of these reports have focused specifically on the development of glucose biosensing schemes where a number of excellent reviews are available[1-4]. The large amount of research activity focused on glucose biosensor development can be attributed to the increasing need for improved diabetes management as well as the idea that glucose represents a well-understood, relatively inexpensive model system for fundamental studies[5]. Within the biosensor research, however, it is relatively rare that the strategies, materials, and constructs shown to be effective for glucose biosensing prove robust and versatile enough to be readily adapted to a dramatically different target molecule. In most cases, such a transition is simply left unexplored. Therefore, a significant development in this area would be the demonstration of a strategy and materials that offer said versatility while maintaining superior performance toward a new target molecule with implications in biomedical application.

Uric acid (UA) is the predominant end product of purine nucleotide catabolism in the human body. UA is present in human blood serum, plasma, urine, and saliva where, due to its poor solubility in biological fluids, it exists predominantly as urate anion at physiological $\mathrm{pH}[6,7]$. For comparison, in aqueous solutions $\left(20-25^{\circ} \mathrm{C}\right)$, the solubility of UA $(\sim 0.0006 \mathrm{~g} / 100 \mathrm{~mL})$ is significantly less than that of glucose $(\sim 90 \mathrm{~g} / 100 \mathrm{~mL})[7]$. UA imbalances in the body have been linked to many diseases. Elevated levels of UA in blood serum are a significant independent predictor for atherosclerotic cardiovascular disease in high-risk individuals[8], peripheral artery disease[9], and chronic kidney disease[10]. Higher concentrations of UA in blood serum have also been shown to be a risk factor for non-alcoholic fatty liver disease[11] and a risk factor for silent brain infarction[12]. Long-standing hyperuricemia also contributes to gout, which is caused by the nucleation and growth of monosodium urate crystals in tissues in and around the joints following the saturation threshold of UA in serum[13]. More recently, UA has also 
been identified as a key biomarker in wounds, indicating the severity of a wound or presence of infection with increasing concentrations[14].

Hyperuricemia is also one of the earliest and most consistent observations noted in pregnancy induced hypertension $(\mathrm{PIH})$, a condition that can lead to a dangerous disorder called pre-eclampsia[15]. PIH-related issues are responsible for a five-fold increase in perinatal mortality, 50,000 world-wide deaths per year, and 15\% of pre-term births in developing countries. The disorder increases risk to both mothers and infants including: shorter gestation periods, smaller birth weights, premature births, and smallfor-gestational-age infants. The current diagnostic for pre-eclampsia risk is blood pressure measurement as well as laboratory-based analyses of blood and urine protein content (i.e., proteinura). While waiting for blood/urine laboratory results PIH can progressively worsen and raise the risk of pre-eclampsia. The currently accepted medical treatment to relieve pre-eclampsia risk is surgical intervention (i.e., cesarean section) on expectant mothers presenting with hypertension and/or other symptoms of PIH[15].

Elevated concentrations of UA have been shown to effectively identify a subset of women with PIH who are at greater risk for maternal or fetal morbidities from preeclampsia. A case study by Roberts et al.[15] examined nearly 1000 pregnancies (19972002) with 400 presenting with hypertension and/or proteinuria. The results of the study showed that the highest patient risk group for pre-eclampsia were those displaying hyperuricemia (i.e., above normal UA levels of $0.5 \mathrm{mM}$ ) even if they did not initially exhibit hypertension or proteinuria. An effective UA biosensor with the potential for in vitro remote sensing or in vivo operation as an intravenous implant with selectivity and sensitivity to UA between 0.1 and $0.6 \mathrm{mM}$ would accelerate risk assessment of pregnancies, identifying higher risk PIH patients, expediting drug therapy and/or surgical intervention - all of which lower perinatal morality rates and reduce risk to mothers as well as lower treatment cost.

UA biosensing schemes based on amperometric detection have been developed using a range of materials including polymer and self-assembled monolayer modified electrodes as well as electrodes incorporating carbon paste or nanomaterials[16, 17]. Key challenges for the electrochemical approach of UA biosensing remain the selectivity of the schemes for UA in the presence of ascorbic acid as well as achieving effective UA 
sensitivity at concentrations directly relevant to the real-time measurements. Enzymaticbased biosensors traditionally target the measurement of a specific by-product of its enzymatic reaction as an indirect measurement of the target substrate. Uricase (UOx)catalyzed reactions of UA:

uric acid $+\mathrm{O}_{2} \stackrel{\text { uricase }}{\longrightarrow}$ allantoin $+\mathrm{H}_{2} \mathrm{O}_{2}$

have been employed with direct electrochemical measurement of $\mathrm{H}_{2} \mathrm{O}_{2}$ product oxidation $[18,19]$ or oxygen depletion ( $1^{\text {st }}$ generation biosensors) $[20]$ as well as the use of mediator species, which react with $\mathrm{H}_{2} \mathrm{O}_{2}$ ( $2^{\text {nd }}$ generation biosensors) for the indirect reporting of UA[21, 22]. Recently, Wang and coworkers reported an elegant "smart bandage" UA biosensor capable of indicating UA release from a wound through the detection of $\mathrm{H}_{2} \mathrm{O}_{2}$ via Prussian blue mediation with a carbon coating[14].

One popular strategy for designing amperometric biosensing schemes remains the modification of electrodes with multiple layers of functional materials. This strategy, known as layer-by-layer (LbL) methodology, represents a critical area of sensor design with several recent reviews available to describe both its historical importance as well as more recent progress and future directions $[23,24]$. A number of different materials have been incorporated into LbL composite films at electrodes for the purpose of sensor development. Layering of polyelectrolyte films with enzymes is an important example of one material used for this purpose, including early work with glucose oxidase[25, 26] as well as later work incorporating nanomaterials into layered polyelectrolyte films[27].

Sol-gels, a gel-like network of polymerized silanes, are another prominent example of a material that has been utilized in LbL design of biosensors with several recent reviews providing an overview summary of the field.[28-30] Sol-gel materials provide a threedimensional scaffolding for multi-layers of encapsulated enzyme at an electrode. The microencapsulation of enzyme within a sol-gel matrix allows for several well-established advantages, including mild synthetic conditions preserving function/structure of the embedded enzymes, chemical inertness, physical rigidity, negligible swelling in aqueous solutions, thermal/photochemical stability, and synthetically tunable porosity, a property of particular interest for biosensing structures[30-33]. Over the years, sol-gels, specifically xerogels formed with solvent evaporation, have been extensively explored in the specific 
context of $1^{\text {st }}$ generation glucose biosensing. For example, Schoenfisch et al. have successfully used layering methodology of sol-gel materials for effective, biocompatible glucose sensors[34]. Others have used layer-by-layer (LbL) approaches with sol-gel materials to introduce nanoparticle networks that enhance the signal from glucose sensors[35]. In the context of UA biosensing, Ferreira et al.[36] and Wang et al.[37] reported layering of UOx within polymer films at indium tin oxide and layering of gold nanoparticle-doped choline at glassy carbon electrodes, respectively.

In this report, we present $\mathrm{LbL}$ construction of a highly effective $1^{\text {st }}$ generation amperometric biosensor for UA. While this study stems from prior, proof-of-concept work with a model glucose biosensing system, it represents a significant research advancement of sensing strategy and technology development that it can be adapted to a clinically relevant target. This study presents unprecedented, systematic characterization and evaluation of each functional layer within the sensing scheme. In establishing, for the first time, a thorough understanding of each material comprising these layers, it is shown that the strategy itself can be optimized toward a specific target species other than the glucose model. The results herein suggest that the general strategy as well as systematic variations of the materials involved may allow for strategic design of schemes flexible enough to be adapted toward a variety of target molecules, thereby spawning the development of additional diagnostic sensors. This study culminates with the development and demonstrated performance of a highly functional UA biosensor that features high sensitivity, effective selectivity, and excellent stability attributes collectively an unmatched combination of performance parameters and a system that maintains the potential to be further developed for in vitro or in vivo operation in PIH risk assessment[15].

\section{Materials and methods}

\subsection{Materials and Instrumentation}


All chemicals were purchased from Sigma-Aldrich unless otherwise specified. Hydrothane (HPU, AL25-80A) and Tecoflex (TPU, SC-80A) polyurethanes (PU) were purchased from AdvanSource Biomaterials and Lubrizol, respectively. All solutions were prepared utilizing $18.3 \mathrm{M} \Omega \cdot \mathrm{cm}$ ultra-purified water $\left(\mathrm{UP} \mathrm{H}_{2} \mathrm{O}\right.$ ). An eight-channel potentiostat (CH Instruments, 1000B) was used to record amperometric current-time (I- $t$ ) curves to evaluate the analytical performance of the sensors, as describe below. Electrochemical cells were comprised of a common $\mathrm{Ag} / \mathrm{AgCl}$ (saturated $\mathrm{KCl}$ ) reference electrode, a common platinum wire counter electrode (Sigma-Aldrich), and either modified platinum or glassy carbon (GCE) working electrodes (3 mm diameter, $\mathrm{CH}$ Instruments). Xerogel structure was assessed using scanning electron microscopy (SEM) imaging (JEOL 6364 SEM) of films deposited on aluminum stubs. PU interfaces were characterized with atomic force microscopy (AFM) (Asylum MFP-3D Bio). Silanes for xerogel fabrication were purchased from Sigma-Aldrich (propyl-trimethoxysilane (PTMS), octyl-trimethoxysilane (OTMS), isobutyl-trimethoxysilane (IBTMS), aminopropyl-trimethoxysilane (APTMS)) or from Gelest, Inc. (hydroxymethyl-triethoxysilane (HMTES)) and were stored in a desiccated glovebox (Plas Laboratories, Inc.) and transported using sealed/desiccated microcentrifuge tubes to exclude moisture until deposition of the material as a film.

\subsection{Optimization and Synthesis of the Uricase Enzyme}

Urate oxidase or uricase (UOx) enzyme from Bacillus fastidiosus ( $\sim 9 \mathrm{U} / \mathrm{mg}$ ) and Candida sp. ( $\geq 2 \mathrm{U} / \mathrm{mg}$ ) were purchased from Sigma Aldrich. Additionally, Bacillus fastidiosus (ATCC strain 29604) UOx gene was obtained from the NCBI database and synthesized by GenScript (Piscataway, NJ) cloned into the NdeI/XhoI site of the pET-21a expression vector. The gene was codon-optimized for expression in an Escherichia coli background prior to synthesis using the optimization tool constructed by Integrated DNA Technologies (Coralville, IA). Plasmids were transformed into chemically competent Rosetta-gami (DE3) cells and were selected on LB agar plates containing ampicillin (100 $\left.\mu \mathrm{g} \mathrm{mL}{ }^{-1}\right)$. Single colonies were incubated overnight at $37{ }^{\circ} \mathrm{C}$ while shaking in $2 \mathrm{XYT}$ broth containing ampicillin $\left(100 \mu \mathrm{g} \cdot \mathrm{mL}^{-1}\right)$. Fresh media was inoculated 1:40 for 
overexpression at $37{ }^{\circ} \mathrm{C}$. Mid-log phase cells were induced with IPTG (0.2 mM) for 3-4 $\mathrm{hr}$. The cells were collected by centrifugation (4,000 rpm, $10 \mathrm{~min})$ and re-suspended in 30 $\mathrm{mL}$ lysis buffer (50 mM phosphate, $\mathrm{pH} 8.0,200 \mathrm{mM} \mathrm{NaCl}, 10 \mathrm{mM}$ imidazole). The cells were then lysed by sonication, and the cell debris was removed by centrifugation $(13,000$ rpm, $25 \mathrm{~min})$. The supernatant was added to a Ni-NTA column for purification by affinity chromatography. UOx was bound to the column via a $\mathrm{C}$-terminal $6 \mathrm{xHis}$ tag and was extensively washed with $20 \mathrm{mM}$ imidizole prior to elution with $250 \mathrm{mM}$ imidizole. At this point, the protein was buffer exchanged into $5 \mathrm{mM}$ phosphate, $\mathrm{pH} 7.0$ and lyophilized (Labconco) overnight for long-term storage at $-20^{\circ} \mathrm{C}$.

\subsection{Fabrication and Characterization of the Xerogel Biosensor}

Platinum working electrodes were successively polished with 1.0, 0.3, and 0.05 $\mu \mathrm{m}$ alumina powder (Electron Microscopy Sciences) and rinsed thoroughly with UP $\mathrm{H}_{2} \mathrm{O}$ prior to electrochemical cleaning via cycling in $0.1 \mathrm{M} \mathrm{H}_{2} \mathrm{SO}_{4}$ between +1.2 and $-0.25 \mathrm{~V}$ at $0.25 \mathrm{~V} / \mathrm{s}$ until the voltammetry was representative of a clean platinum surface. Xerogel layering and construction of the biosensing components was performed with an adaptation to prior procedures[5]. Typically, the process, performed inside a humiditycontrolled chamber (Cole-Parmer, 50\% RH), involved separate mixtures of $4 \mathrm{mg}$ of UOx in $75 \mu \mathrm{L}$ of UP water and $25 \mu \mathrm{L}$ of silane in $100 \mu \mathrm{L}$ of tetrahydrofuran (THF) being agitated (Vortex) for 10 minutes. A $50 \mu \mathrm{L}$ aliquot of the UOx solution was added to the $\mathrm{THF} /$ silane mixture and agitated for an additional 10 minutes. A $3 \mu \mathrm{L}$ aliquot of the resulting sol-gel solution was deposited on the platinum electrode and allowed to dry at $50 \% \mathrm{RH}$ for $\sim 8$ minutes (i.e., enzyme-doped xerogel layer). A second layer of sol-gel without UOx (i.e., diffusion-limiting xerogel layer), was prepared and deposited in identical fashion and placed on top of the first sol-gel layer. The sol-gels were aged at $50 \% \mathrm{RH}$ for 48 hours to form xerogels. Note: The environment during aging is of crucial importance, since sensor performance has been shown to vary drastically depending on time and $R H[5,35]$. SEM and electrochemical characterization of the xerogel layers has been either previously presented[5, 35] or is included in Supplementary Material herein. Additionally, prior work in the lab evaluated the porosity and surface area of drop-cast 
xerogel films, including films from silane precursors used in this report, using electrochemistry, spectroscopy, and contact angle goniometry.[5]

In order to apply the 1:10 polyluminol:polyaniline (PL-A) layer, a previously discovered procedure was used that is known to provide selectivity for UA sensing[38, 39]. The xerogel-modified electrode described above was immersed in $25 \mathrm{~mL}$ stirred solution of a $0.5 \mathrm{mM}$ luminol (saturated) and $5 \mathrm{mM}$ aniline solution mixture $(0.1 \mathrm{M}$ $\mathrm{H}_{2} \mathrm{SO}_{4} ; \mathrm{N}_{2}$ degassed, $20 \mathrm{~min}$.). The layer was electropolymerized while stirring using cyclic voltammetry at a sweep cycle of $50 \mathrm{mVs}^{-1}$ cycling between 0.0 and $1.0 \mathrm{~V}$ (vs. $\mathrm{Ag} / \mathrm{AgCl}$ reference electrode) for 12 cycles. Electrodes were rinsed with UP water and left to dry (ambient, 10 min.).

The outer polyurethane (PU) layer semipermeable membrane was applied as previously reported[5, 34, 35]. Briefly, PU blends were prepared from dissolving different ratios of HPU and TPU in ethanol and THF, which was stirred overnight. A 10.0 $\mu \mathrm{L}$ aliquot of the PU blend was then deposited on the bare or xerogel modified electrode and allowed to dry (ambient, $30 \mathrm{~min}$.).

\subsection{Evaluation of Sensor Performance}

As in previous work $[5,34,35]$, assembled sensors were pre-treated by being soaked in PBS $(65.55 \mathrm{mM}$; $\mathrm{pH}=7)$ for 1 hour to ensure saturation of the xerogel matrix. The sensors then were immersed in $25 \mathrm{~mL}$ of stirred PBS $(65.55 \mathrm{mM}$; $\mathrm{pH}=7)$ for amperometric measurements wherein the working electrode potential was held at $+0.65 \mathrm{~V}$ (vs. $\mathrm{Ag} / \mathrm{AgCl}$, satrd' $\mathrm{KCl}$ ) for $20 \mathrm{~min}$ before successive $25 \mu \mathrm{L}$ injections of $50 \mathrm{mM}$ UA solution at $200 \mathrm{~s}$ intervals and the I- $t$ curve was collected. Sensor sensitivity to UA was determined via linear regression analysis of calibration curves for the sensor current response at increasing UA concentrations. As in previous studies[5, 34, 35], a conservative estimate of sensor response time $\left(t_{r-95 \%)}\right)$, time before $95 \%$ of total response recorded, was employed. As previously described[5, 34, 35], film permeability was measured via permeability indices (PI) using the following equation: 
$\mathrm{PI}=\frac{\Delta \mathrm{I}_{\text {film }}}{\Delta \mathrm{I}_{\text {bare }}} \times 100$

where $\Delta \mathrm{I}_{\text {film }}$ and $\Delta \mathrm{I}_{\text {bare }}$ are the measured changes in current for a species at a filmmodified and bare electrode, respectively. As previously described in biosensing literature, amperometric selectivity coefficients $\left(\mathrm{K}^{a m p}\right)$ were used to evaluate interferent responses and were calculated using the following equation:

$\mathrm{K}_{\mathrm{UA}, \mathrm{j}}^{\mathrm{amp}}=\log \left(\frac{\Delta \mathrm{I}_{\mathrm{j}} / \mathrm{C}_{\mathrm{j}}}{\Delta \mathrm{I}_{\mathrm{UA}} / \mathrm{C}_{\mathrm{UA}}}\right)$

where $\Delta \mathrm{I}_{\mathrm{j}}$ and $\Delta \mathrm{I}_{\mathrm{UA}}$ are the measured currents for a specific interferent species $(\mathrm{j})$ and UA at concentrations of $\mathrm{C}_{\mathrm{j}}$ and $\mathrm{C}_{\mathrm{UA}}$, respectively[34, 35, 40]. If necessary, sensors were stored at $4-7^{\circ} \mathrm{C}$ immersed in PBS (65.55 mM; pH 7).

\section{Results and discussion}

The general design of the layer-by-layer (LbL) biosensing scheme originally developed and demonstrated for a glucose sensing model system[5] is depicted in Scheme I and features four functional layers modifying a platinum electrode: (1) an enzyme-doped xerogel layer; (2) a diffusion-limiting xerogel layer; (3) an inner selective electropolymer layer; and (4) an outer-selective polyurethane layer. The enzymatic reaction occurs in the lowest layer, the enzyme-doped xerogel, where peroxide is produced and oxidized at the electrode, indirectly indicating the presence of the substrate species. This specific combination of layering was used to successfully create an effective glucose sensor and prompted strategic exploration adapting the scheme to other targets of interest, in this case, UA biosensing. With the exception of the electropolymer layer, which is specifically developed elsewhere[39], each material comprising the layers was systematically investigated and optimized to UA detection. Beyond layer optimization, composite films were evaluated on sensing performance, including sensitivity, response time, selectivity, stability, and shelf-life. 


\subsection{Outer Selective Polyurethane Membrane}

The semi-permeable polyurethane layer (PU) controls the diffusion of analyte (i.e., UA) and oxygen into the composite film, while also providing some selectivity based on its hydrophobic character[34]. The PU mixture used in previous sensing glucose biosensing schemes was a 50:50 blend of hydrophilic polyurethane (HPU) and hydrophobic polyurethane (TPU) [5, 34]. To specifically evaluate and optimize the PU for UA biosensing, however, several experiments were conducted on a series of electrodes coated with different ratios of HPU:TPU blends: 100:0, 75:25, 50:50, 25:75, and 0:100. Voltammetry and amperometry were used to assess the PU layer's hydrophobic and blocking characteristics as well as its permeability to $\mathrm{H}_{2} \mathrm{O}_{2}$ and UA - components critical to the proposed sensing scheme.

Observational experiments probing the PU interface hydrophobicity and porosity utilized cyclic voltammetry of a potassium ferricyanide $(\mathrm{FeCN})$ at each film type. UA itself, while electroactive, exhibits voltammetry not pronounced enough at bare or modified platinum electrodes for direct assessment at most modified electrodes[41]. FeCN voltammetric probing proved an effective tool to learn about the electrode interfaces. Figure 1A displays FeCN voltammetry at various PU films on platinum compared to that at a bare platinum electrode. The addition of any PU layers significantly decreases the voltammetric signature of $\mathrm{FeCN}$, exhibiting drastically lower current. A closer look at the voltammetry at the PU films (Fig. 1A, inset) reveals a significant trend whereby voltammetry of $\mathrm{FeCN}$, acting as an UA mimic, is most significant at the 100\% HPU interface (Fig. 1A, b), subsequently fades as the hydrophobic (TPU) component is increased (Fig. 1A, b $\rightarrow$ e), and is completely blocked at the most hydrophobic, 100\% TPU interface (Fig. 1A, f). These results suggest that the PU layer most conducive to UA sensing would be the $100 \%$ HPU coating, which also shows a substantial difference in the morphology from the $100 \%$ TPU according to SEM and AFM microscopy (Supplementary Material).

Identical PU interfaces were also tested for amperometric response toward $\mathrm{H}_{2} \mathrm{O}_{2}$. PU-coated electrodes and a bare platinum electrode were held at $+0.65 \mathrm{~V}$ during 
successive injections of $\mathrm{H}_{2} \mathrm{O}_{2}$, generating current-time (I- $t$ ) curves. The results (Figure 1B) reiterate the $\mathrm{FeCN}$ voltammetry findings that with the addition of any PU layer, the overall signal is diminished but the most hydrophilic layer, 100\% HPU (Fig. 1B,b), allows for the most substantial amount of $\mathrm{H}_{2} \mathrm{O}_{2}$ oxidation. Figure $1 \mathrm{C}$ summarizes $\mathrm{FeCN}$ voltammetry anodic peak current $\left(\mathrm{i}_{\mathrm{p}, \mathrm{a}}\right)$ and amperometric peak current for $\mathrm{H}_{2} \mathrm{O}_{2}$ oxidation $\left(i_{\text {ox }}\right)$ at the different PU layers, showing that as the percentage of HPU increases in the PU film, the average current in both cases also increases. It noteworthy that the overall, general blockage of $\mathrm{H}_{2} \mathrm{O}_{2}$ by any PU layer may actually be beneficial since it assists in confining $\mathrm{H}_{2} \mathrm{O}_{2}$ near the electrode, acting as a barrier to diffusion away from the interface that would result in subsequent loss of signal.

The most significant role of the outer selective PU membrane is the amperometric current generated via direct injections of UA as a function of the composition of HPU versus TPU in a modified electrode poised at $+0.65 \mathrm{~V}$. Since UA is electroactive, any signal generated from its direct oxidation is due to the molecule gaining access to the electrode through the PU layer. UA amperometry at various PU modified electrodes should reveal the most conducive PU combination for UA to permeate the PU layer (Figure 2). Results again show that with any PU modification there is significant UA electrochemistry blockage, but the observed amperometric response is greatest with PU layers possessing higher HPU components.

As an additional evaluation of the PU membrane, permeability of UA, common endogenous interferents (glucose, oxalic acid, sodium nitrite), and artificial interferents (acetaminophen, ascorbic acid) was assessed. In this study, glassy carbon electrodes (GCE), which exhibit greater voltammetric response toward UA than platinum electrodes, were implemented to isolate effective evaluation of the PU layer[39]. GCEs were modified with different blends of PU were subjected to amperometry $(+0.65 \mathrm{~V})$ with injections of UA and the aforementioned interferent species. Permeability indices or PI (see Experimental Details) were calculated for each species and found to be negligible for some interferents (i.e., oxalic acid, glucose, and sodium nitrate yielded no significant amperometric response at any of the films). PI values for acetaminophen, ascorbic acid, and UA as well as ratios for UA-to-acetaminophen and UA-to-ascorbic acid PI values were also tabulated (Supplementary Material). The results again suggest 
higher HPU content in the PU films (75:25 and 100:0) are most conducive for UA signal and provide reasonable interferent discrimination. Based on the collective findings of these experiments, the PU layer was optimized for UA sensing implementing $100 \% \mathrm{HPU}$ as the outer selective membrane.

\subsection{Inner Selective Electropolymer Membrane}

The electropolymer layer serves as a critical semi-permeable membrane, providing selectivity through common interferent discrimination. As mentioned previously, the systematic design and identification of the electropolymerized layer utilized for the LbL UA sensing strategy was extensively developed and reported elsewhere[39]. An effective polymer layer providing selectivity against interferents (e.g., ascorbic acid, sodium nitrite, glucose, and oxalic acid) was found to be a 1:10 mixture of polyluminol-to-polyaniline. As described in the Experimental Section, the polyluminolpolyaniline (PL-A) layer is electropolymerized on the modified electrodes following xerogel bi-layer (see below) formation.

\subsection{Xerogel Bi-layer}

The xerogel bi-layer consists of two distinct xerogel layers: (a) layer doped with enzyme (designated with an * in certain figures/tables presented herein) housing the enzymatic reaction producing $\mathrm{H}_{2} \mathrm{O}_{2}$ and; (b) xerogel layer not doped with enzyme acting as a diffusion-limiting layer. This type of bi-layer is depicted and detailed in the enlargement insets of Scheme I. Bi-layer designs of this nature have demonstrated effective functionality as seen in prior findings from this lab and others[5, 34]. It should be noted that physical characterization of different xerogel materials (i.e., gels formed formed from varying silane precursors), including SEM and electrochemical characterization as well as porosity/surface area assessment, have been performed prior to this study[5, 35] or are included as part of the Supplementary Material in this report.

As an initial study of the xerogel layers, the first layer housing the UOx enzyme was examined. Specifically, the influence of UOx enzyme species toward UA sensitivity 
within an octyl-trimethoxysilane (OTMS) xerogel a matrix previously determined to be suitable for these schemes, was investigated. Sensors were constructed with different species of UOx in the first layer of OTMS followed by a second layer OTMS without UOx and capped with PU (100\% HPU). To evaluate UOx activity and sensitivity, the inner selective electropolymer film was not applied in these systems as its primary purpose is selectivity. Three types of sensors were created, each featuring a different UOx species, including Bacillus fastidiosus and Candida sp, as well as, Bacillus fastidiosus produced in-house from codon-optimized expression in Escherichia coli. Each system was subjected to successive injections of UA standard while the amperometric I- $t$ response was recorded (not shown) and translated into calibration curves (Figure 3A) with acceptable linear range. The results suggest that the in-house optimized Bacillus fastidiosus enzyme is able to achieve significant sensitivity (i.e., slope) and defined step responses across the relevant UA concentration range (i.e., 100$600 \mu \mathrm{M})$ at a significantly lower operating cost compared to commercially available sources of the enzyme. Similar calibration curves for systems featuring each of the enzyme species embedded in xerogels with and without PU capping layer were achieved as well (see Supplementary Materials). Based on these comparisons, the remainder of the study was conducted with the in-house Bacillus fastidiosus UOx.

The loading (i.e., the doping level) of UOx within the first xerogel layer was explored. Composite films of OTMS xerogel bi-layers with and without the PU capping layer were formed with different amounts $(3,6$, and $9 \mathrm{mg})$ of UOx doped into the first xerogel layer and their amperometric response to successive UA injections was monitored. The results suggest that greater UOx concentrations were not beneficial to performance (Supplementary Material). That is, UA sensitivity was not improved with higher UOx doping levels in the xerogel. As such, subsequent UOx-doped xerogels in the study were prepared with only 3-4 mg of UOx. In enzyme-based biosensing schemes, it is thought that higher concentrations of certain enzymes permit the rate of substrate conversion to become independent of enzyme concentration[34]. The linearity of the results of the calibration curves, both in testing the enzyme species and loading as well as in subsequent calibration curves to be presented, suggests that the 3-4 mg UOx doping level within the xerogels represents an excess of available UOx and allows the system to 
avoid saturation effects that cause non-linearity and calibration curve plateau which would necessitate Michaelis-Menton kinetic measurements[42, 43]. Additionally, the linear response overlaps well with the relevant physiological concentration range of UA, suggesting that UOx activity is not a factor in these sensing schemes. Similar linearity is observed in all subsequent measurements of LbL assemblies that show promise for UA sensor development.

Ionic strength $(\mu)$ of the sample matrix is particularly important to evaluate given the goals of eventually using these findings toward in-vitro, remote bedside sensors or invivo implantable devices for continuous UA monitoring. Electrochemical sensing is dependent on solution activity rather than simple concentration, making evaluation of the sensor at biological levels of $\mu$ an important facet of the sensor development. OTMS bilayer systems were used to evaluate $\mu$ dependence of the sensor response to UA, again without incorporating the selectivity-based electropolymer layer. Calibration curve data for UA response of the Pt/OTMS*/OTMS/PU system was collected at different concentrations of phosphate buffer solution (4.4-250 mM PBS at $\mathrm{pH}$ 7) which differ significantly in ionic strength as well $(10<\mu<572 \mathrm{mM})$ - see Supplementary Material. The UA sensitivity of the composite film seems to increase with $\mu$ to a point $(65.55 \mathrm{mM}$; $\mu=150 \mathrm{mM}$ ) before plateauing at even higher values (Supplementary Material). Given the sensitivity found at $65.55 \mathrm{mM}$ PBS $(\mu=150 \mathrm{mM})$ and its similarity to the $\mu$ of blood plasma (145 mM)[44], all subsequent experiments were conducted in $65.55 \mathrm{mM}$ PBS.

Optimization of enzyme species, loading, and ionic strength, prompted investigation into the silane precursor utilized in forming the xerogel layers. It should be noted that the sensor designs discussed herein utilize xerogel bi-layers derived from the same silane precursor - a homogeneous xerogel bi-layer. Heterogeneous xerogel bi-layer systems, where each layer of the xerogel is derived from a different silane precursor, were extensively studied in the glucose model system and showed convincing results that no gain in either sensitivity or selectivity could be achieved by using different types of xerogels in the same composite film (Supplementary Material). Homogeneous bi-layer systems were therefore the only systems further explored for UA biosensing

Based on prior evaluation and reporting of silane precursors for xerogel formation[5], a subset of silane precursors, including HMTES, OTMS, IBTMS, PTMS, 
and APTMS, were identified for UA biosensing development. Xerogel bi-layers from these precursors were formed at GCEs and their amperometric responses toward UA and common interferents were again translated to PI values (Supplementary Material). All five types of xerogel by-layers showed relatively high PI indices for UA, acetaminophen, and ascorbic acid with negligible values recorded for other interferent species.

Homogeneous xerogel bi-layers at platinum electrodes capped only with PU (i.e., no electropolymer layer) were also constructed from these silane precursors to isolate and evaluate the effect of different xerogels on UA sensitivity. The amperometric response of each of the modified electrodes during successive injections of UA (I- $t$ curve) was recorded (not shown) and calibration curves derived from the responses of the different sensors are shown in Figure 3B. An excellent stair-step response, indicated with solid symbols in the curves, was achieved for all xerogel systems. This result is consistent with prior research using layered composite films for glucose sensing. Most of the films (HMTES, OTMS, IBTMS and PTMS) showed effective sensitivity toward UA. PI values for these bi-layer films capped with 100\% HPU at GCEs reiterated significant and increasing permeability for ascorbic acid, UA, and acetaminophen (Supplementary Material). This result made increased selectivity one of the primary goals of the fully layered composite films.

\subsection{Uric Acid Sensing Performance - Complete System}

With each layer optimized for UA permeability and sensitivity, performance of the complete sensor (i.e., xerogel bi-layer, electropolymer membrane, and PU capping layer) was evaluated. Sensor sensitivity, selectivity, response time, stability, and shelflife were assessed for the most promising schemes: xerogel bi-layers of HMTES, OTMS, IBTMS, and PTMS capped with both inner selective PL-A, and outer selective 100\% HPU membranes (Scheme I). Figure 4A shows the calibration curves of all four systems with comparable sensitivity toward UA $(\sim 0.6-1.0 \mathrm{nA} / \mu \mathrm{M})$, a result paralleling the previous systems tested without the PL-A electropolymer layer (Fig. 3B). All of the systems maintained excellent linear ranges with stair-step responses across the physiologically relevant range of UA concentration (100 to $700 \mu \mathrm{M}$ linear and dynamic 
ranges). In addition, and with the exception of the film using PTMS, all showed average response times of $\leq 10$ seconds toward UA. All of the films showed very low limits of detection (LOD) as well though, in this case, the parameter is less critical than analyte sensitivity within the physiologically relevant range. A complete table summary of the performance properties for the four complete systems is provided (Table 1) and a comparison of these properties to other literature reports of uric acid biosensors is provided in the Supplementary Materials as well.

A critical biosensor characteristic is selectivity for the targeted analyte in the presence of common interferents. While the use of an enzymatic reaction allows for a certain level of natural selectivity[1], some species can still be problematic particularly given the relatively high potential used to oxidize $\mathrm{H}_{2} \mathrm{O}_{2}$. In the case of UA biosensing, acetaminophen, ascorbic acid, sodium nitrite, oxalic acid, and glucose are considered interferents[16]. Figure 4B provides amperometric I- $t$ curves during injections of UA and common interferents at the full HMTES xerogel system which was determined to give the highest UA sensitivity (Fig. 4A). Acetaminophen yielded the only significant response while UA exhibited concentration sensitive responses $(100 \mu \mathrm{M}$ and $300 \mu \mathrm{M}$ UA, Fig. 4B) in the presence of the interferents. This qualitative selectivity assessment from I- $t$ curves can be quantitatively described by measuring selectivity coefficients for each interferent compared to UA (Experimental Details) [5, 34]. Figure 4C displays the comparison of selectivity coefficients for the HMTES, OTMS, IBTMS and PTMS full sensors. Positive coefficients indicate that selectivity for a specific species while negative coefficients suggest the species is sufficiently discriminated against. Two key findings are suggested by the results. First, while all the systems give effective sensitivity toward UA, the HMTES and OTMS systems were most UA selective. Second, while the full composite film effectively discriminates against most common intererents, acetaminophen, a pain-relieving synthetic drug that is commonly prescribed as safe for pregnant women[45], remains problematic and is discussed further below.

Figure 5 shows the results of a more focused investigation of the HMTES full sensing system, determined to be the most effective optimized system tested in terms of both UA sensitivity and interferent discrimination (Fig. 4). Fig. 5A displays the I- $t$ curve and corresponding calibration curve (Fig. 5A, inset) during successive UA injections for 
the HMTES full system. Interestingly, the layered composite films display sensing effectiveness only when all four layers are present. A LbL examination of the I- $t$ response and calibration curves, Fig. 5B and 5C, respectively, suggest that it is the layers working in concert with each other that allow the observed sensing performance. While the addition of layers dampens the overall signal, the stair-step response with relatively little noise is readily apparent and measurable at relevant concentrations. A similar phenomenon is observed with full system HMTES selectivity testing - effective interferent discrimination only with all four layers (Figure 6). Analogous selectivity trends were obtained with the OTMS and IBTMS full systems as well (Supplementary Material). APTMS and PTMS systems were not further tested due to inferior sensitivity and response time, respectively.

It is clear from the results of Figs. 5 and 6 that the layers work in concert with each other to achieve the observed sensing performance at the expense of signal magnitude. That is, the full system has the lowest current response for UA (Fig. 5C) largely because the final two ad-layers (i.e., outer xerogel diffusion-limiting layer and outer polyurethane layers) provide a diffusional limitation toward interfering species at the expense of UA signal. While dampening the signal, these layers provide physical stability and electrode-to-electrode consistency, insulating the current response from variations in enzyme loading. These layers appear to regulate the diffusion of UA substrate to the enzyme as evidenced by the absence of saturation behavior in the calibration curves over the targeted concentration range, a result suggesting mixed kinetic and diffusional control of the signal production[42]. Strategies for enhancing the magnitude of the signal in these film systems are currently being explored.

For a sensing model to be effective stability during use and shelf-life during storage must be considered. Sensors should maintain stability (sensitivity, response time) and selectivity toward the analyte over a period of time relevant to its intended application. These UA sensors were monitored for ten days, significantly longer than typical pre-birth stays of PIH-suspected pregnant mothers. Figure 7 tracks the sensitivity, response time, and selectivity coefficients for the HMTES full systems over the 10 days. Results suggest little loss of sensitivity, mild fluctuations in response time, 
and sustained selectivity for UA. Similar results were observed for the OTMS and IBTMS systems as well (Supplementary Material).

Similarly, the shelf-life of the sensors was tested. In these experiments, numerous sensors were created and tested in a single day (day 0 ) before being stored for a maximum of 30 days. At regular intervals, different sets of stored sensors were tested for sensitivity, response time, and selectivity. Figure 8 illustrates the trends of these performance parameters as a function of sensor storage time where sensitivity is largely maintained, there is a reasonable fluctuation of response time ( $<25$ seconds), and virtually no degradation of selectivity coefficients during storage up to 30 days.

A detailed list of sensing performance parameters for the UA biosensor developed in this study compared with literature reports of different types of UA sensors is provided in Supplementary Material. It should be noted that these reports do not always measure the same parameters (e.g., sensitivity [36]) and/or provide descriptions of how other parameters are measured. In particular, for example, the methodology of response time measurements is often not reported whereas our response times represent conservative estimates of this parameter (Experimental Details). Also, in addition to other $1^{\text {st }}$ generation sensors, this biosensor's performance was compared to other types of sensors featuring different mechanisms including direct electrochemical detection of UA, fluorescence and oxygen depletion measurements, as well as $2^{\text {nd }}$ generation biosensing schemes. It is important to note that even though some of these other types of sensors perform similarly, their respective mechanisms may not be as advantageous toward further development such as miniaturized, in-vivo devices, for example. Such limitations include spectroscopic-based detection challenges within flesh, the variability of oxygen levels in biological systems, as well as the complexity/danger of utilizing freely diffusing electron transfer mediator species in $2^{\text {nd }}$ generation biosensors[1-4]. Some of the $1^{\text {st }}$ generation UA sensors reported also failed to have a linear response for physiological concentrations of $\mathrm{UA}[22,37]$. Thus, while a literature comparison is appropriate, direct comparison and judgment of only the listed parameters should be weighed with the other aforementioned aspects of sensor attributes.

In comparing this $1^{\text {st }}$ generation UA biosensor's performance to the literature reports, it equals or exceeds measured performance parameters of other sensors 
(Supplementary Material). Within this comparison, some sensors reported slightly shorter response times without describing how the measurement was made. Indeed, few examples of UA sensor in the literature are comparable to this biosensor if one considers the combination of all regularly measured/reported performance parameters: sensitivity, response time, linear/dynamic range, limit of detection, and stability and the additional aspects studied here. For example, the current study examines, in detail, the selectivity against common interferents. This is an important sensing parameter that was not even referenced in some studies[22] or showed poor discrimination against ascorbic acid in others[20], an interferent completely eliminated in the biosensor presented here. While acetaminophen interference does remains an issue that we continue to investigate, it can be practically managed during UA monitoring with the sensor in conjunction with recording accurate patient history to determine drug ingestion six hours prior to measurement[46]. The current study also includes an assessment of the UA biosensor shelf-life, a property not addressed in any of the comparison literature.

\section{Conclusions}

This study represents an unprecedented and detailed evaluation of the functionality of each material in a LbL biosensing scheme and presents a viable UA sensor that may be later miniaturized into biomedical devices for in vivo implantation or real-time in-vitro measurement for $\mathrm{PIH}$ detection/monitoring $[47,48]$. The functional layering of UOx-doped and un-doped xerogel material, the selective electropolymerized PL-A, and optimized PU membrane work in concert as an effective UA biosensor that is poised for further development, perhaps incorporated on an intravenous wire/needle electrode, for example[34, 49]. A second aspect of this study to note is the successful adaptation of the LbL xerogel-based sensing scheme developed from the glucose model system $[5,34,35]$ to a new and separate target molecule of clinical relevance[15]. The presented work suggests that each layer serves a function and can be methodically optimized toward target species. In context, the work seemingly provides a broad spectrum biosensing strategy able to be deliberately applied to specific biomedical needs 
in the area of diagnostic tools that can save time, lower expense, and maintain patient health (e.g., cholesterol[50] and galactose sensor development[51].

\section{Acknowledgements}

This research was generously supported by funding from the National Science Foundation (CHE-1401593), Virginia's Commonwealth Health Research Board, and the Beckman Foundation (MJP) as well as student fellowships from the University of Richmond's Undergraduate Research Committee (GEC, RHL). We would like to thank Nick Poulos, Jackson Hall, Kaiwen Chen, Aastha Minocha, Microscopy Director Christie Davis, and Dr. Matthew Trawick (AFM) for their important contributions to this work. We gratefully acknowledge the following people for making research possible at the University of Richmond: Drs. T. Leopold, R. Kanters, D. Kellogg, R. Miller, andR. Coppage, as well as Russ Collins, Phil Joseph, Mandy Mallory, and Lamont Cheatham.

\section{Supplementary Materials}

AFM/SEM and permeability studies of PU layer; Amperometric I- $t$ and calibration curve for different UOx species and loading levels in xerogel; ionic strength dependence; Amperometric I-t, calibration curve, selectivity coefficient, response time, stability comparisons for OTMS and IBTMS systems; Shelf-life studies with HMTES; Literature comparison of UA biosensor performance parameters; Study of heterogeneous xerogel layer system (cyclic voltammetry, SEM, amperometric I- $t$, calibration curve, permeability, and selectivity coefficient results).

\section{AUTHOR INFORMATION}

Corresponding Author 
*Email: $\underline{\text { mleopold@ richmond.edu }}$

Notes: The authors declare no competing financial interest.

\section{References}

[1] J. Wang, Electrochemical glucose biosensors, Chemical Reviews. 108 (2008) 814-825.

[2] J. Wang, In vivo glucose monitoring: Towards 'Sense and Act' feedback-loop individualized medical systems, Talanta. 75 (2008) 636-641.

[3] A. Heller, B. Feldman, Electrochemical glucose sensors and their applications in diabetes management, Chem. Rev. 108 (2008) 2482-2505.

[4] B.J. Privett, J.H. Shin, M.H. Schoenfisch, Electrochemical Sensors, Anal. Chem. 82 (2010) 4723-4741.

[5] N. Poulos, J. Hall, M. Leopold, Functional layer-by-layer design of xerogel-based first generation amperometric glucose biosensors, Langmuir 31 (2015) 1547-1555.

[6] S.A. Fathallah-Shaykh, M.T. Cramer, Uric acid and the kidney, Pediatr Nephrol. 29 (2014) 999-1008.

[7] K. Inoue, T. Namiki, Y. Yoshimura, H. Nakazawa, Determination of uric acid in human saliva by high-performance liquid chromatography with amperometric electrochemical detection, J. Chromatogr. B. 785 (2003) 57-63.

[8] P. Strazzullo, J.G. Puig, Uric acid and oxidative stress: Relative impact on cardiovascular risk, Nutr. Metab. Carbiovasc. Dis. 17 (2007) 409-414.

[9] A. Shankar, B.E.K. Klein, F.J. Nieto, R. Klein, Association between serum uric acid level and peripheral arterial disease, Atherosclerosis. 196 (2008) 749-755.

[10] M. Chonchol, M.G. Shilipak, R. Katz, M.J. Sarnak, A.B. Newman, D.S. Siscovick, B. Kestenbaum, J.K. Carney, L.F. Fried, Relationship of uric acid with progression of kidney disease, Am. J. Kidney Dis. 50 (2007) 239-247.

[11] Y. Li, C. Xu, C. Yu, L. Xu, M. Miao, Association of serum uric acid level with nonalcoholic fatty liver disease: A cross-sectional study, J. Hepatol. 50 (2009) 1029-1034.

[12] S.H. Heo, S.H. Lee, High levels of serum uric acid are associated with silent brain infarction, J. Neurol. Sci. 297 (2010) 6-10. 
[13] R.A. Terkeltaub, Gout, N. Engl. J. Med. 349 (2003) 1647-1650.

[14] P. Kassal, J. Kim, R. Kumar, W.R. de Araujo, I.M. Steinberg, M.D. Steinberg, J. Wang, Smart bandage with wireless connectivity for uric acid biosensing as an indicator of wound status, Electrochem. Commun. 56 (2015) 6-10.

[15] J.M. Roberts, L.M. Bodnar, K.Y. Lain, C.A. Hubel, N. Markovic, R.B. Ness, R.W. Powers, Uric acid is as important as proteinura in identifying fetal risk in women with gestational hypertension, Hypertension. 46 (2005) 1263-1269.

[16] P.E. Erden, E. Kılıç, A review of enzymatic uric acid biosensors based on amperometric detection, Talanta. 107 (2013) 312-323.

[17] J.S.N. Dutt, M.F. Cardosi, C. Livingstone, J. Davis, Diagnostic Implications of Uric Acid in Electroanalytical Measurements, Electroanal. 17 (2005) 1233-1243.

[18] F. Arslan, An Amperometric Biosensor for Uric Acid Determination Prepared from Uricase Immobilized in Polyaniline-polypyrrole Films, Sensors. 8 (2008) 5492-5500.

[19] N. Chauhan, C.S. Pundir, An Amperometric Uric Acid Biosensor Based on Multiwalled Carbon Nanotube-Gold Nanoparticle Composite, Anal. Biochem. 413 (2011) 97-103.

[20] Y. Zhang, G. Wen, Y. Zhou, S. Shuang, C. Dong, M.M.F. Choi, Development and analytical application of an uric acid biosensor using an uricase-immobilized eggshell membrane, Biosens. Bioelectron. 22 (2007) 1791-1797.

[21] T. Ahuja, V.K. Tanwar, S.K. Mishra, D. Kumar, A.M. Biradar, Rajesh, Immobilization of uricase enzyme on self-assembled gold nanoparticles for application in uric acid biosensor, J. Nanosci. Nanotechnol. 11(6) (2011) 4692-4701.

[22] T. Nakaminami, S. Ito, S. Kuwabata, H. Yoneyama, Uricase-catalyzed oxidation of uric acid using an artificial electron acceptor and fabrication of amperometric uric acid sensors with use of a redox ladder polymer, Anal. Chem. 71 (10) (1999) 1928-1934.

[23] K. Ariga, Y. Yamauchi, G. Rydzek, Q. Ji, Y. Yonamine, K.C.-. Wu, J.P. Hill, Layer-by-layer nanoarchitectonics: Invention, innovation, and evolution, Chem. Lett. 43 (2014) 36-68.

[24] G. Rydzek, Q. Ji, M. Li, P. Schaaf, J.P. Hill, F. Boulmedais, K. Ariga, Electrochemical nanoarchitectonics and layer-by-layer assembly: From basics to future, Nano Today 10 (2015) 138-167.

[25] M. Onda, Y. Lvov, K. Ariga, T. Kunitake, Sequential actions of glucose oxidase and peroxidase in molecular films assembled by layer-by-layer alternate adsorption, Biotechnol. Bioeng. 51 (1996) 163-167.

[26] M. Onda, K. Ariga, T. Kunitake, Activity and Stability of Glucose Oxidase in Molecular Films Assembled Alternately with Polyions, J. Biosci. Bioeng. 87 (1999) 69-75. 
[27] A.R. Schmidt, N.D.T. Nguyen, M.C. Leopold, Nanoparticle film assemblies as platforms for electrochemical biosensing: Factors affecting the amperometric signal enhancement of hydrogen peroxide, Langmuir. 29 (2013) 4574-4583.

[28] A. Walcarius, M.M. Collinson, Analytical Chemistry with Silica Sol-Gels: Traditional Routes to New Materials for Chemical Analysis, Annual Review of Analytical Chemistry. 2 (2009) 121-143.

[29] R. Gupta, Bioactive materials for biomedical applications using sol-gel technology, Biomedical materials (Bristol). 3 (2008) 034005.

[30] R. Pauliukaite, Development of electrochemical biosensors based on sol-gel enzyme encapsulation and protective polymer membranes, Analytical and bioanalytical chemistry. 390 (2008) 1121-1131.

[31] U. Narang, P.N. Prasad, F.V. Bright, K. Ramanathan, N.D. Kumar, B.D. Malhotra, M.N. Kamalasanan, S. Chandra, Glucose biosensor based on a sol-gel-derived platform, Anal. Chem. 66 (1994) 3139-3144.

[32] S. Liu, Y. Sun, Co-immobilization of glucose oxidase and hexokinase on silicate hybrid solgel membrane for glucose and ATP detections, Biosensors and Bioelectronics. 22 (2007) 905911.

[33] V.B. Kandimalla, V.S. Tripathi, H. Ju, Immobilization of biomolecules in sol-gels: Biological and analytical applications, Crit. Rev. Anal. Chem. 36 (2006) 73-106.

[34] A. Koh, Y. Lu, M.H. Schoenfisch, Fabrication of nitric oxide-releasing porous polyurethane membranes-coated needle-type implantable glucose biosensors, Anal. Chem. 85 (2013) 1048810494.

[35] M.H. Freeman, J.R. Hall, M.C. Leopold, Monolayer-protected nanoparticle doped xerogels as functional components of amperometric glucose biosensors, Anal. Chem. 85 (2013) 40574065 .

[36] M.L. Moraes, U.P. Rodrigues Filho, O.N.J. Oliveira, M. Ferreira, Immobilization of uricase in layer-by-layer films used in amperometric biosensors for uric acid, J. Solid State Electrochem. 11 (2007) 1489-1495.

[37] P. Wang, Y. Li, X. Huang, L. Wang, Fabrication of layer-by-layer modified multilayer films containing choline and gold nanoparticles and its sensing application for electrochemical determination of dopamine and uric acid, Talanta 73 (2007) 431-437.

[38] E. De Robertis, R.S. Neves, A.J. Motheo, Electropolymerization studies of PAni/(poly)luminol over platinum electrodes, Mol. Cryst. Liquid Cryst. 484 (2008) 688-700.

[39] K. Chen, G.E. Conway, G.A. Hamilton, M.L. Trawick, M.C. Leopold, Electro-polymerized layers as selective membranes in first generation uric acid biosensors, J. Applied Echem. 46 (2016) 603-615. 
[40] G. Sánchez-Obrero, M. Cano, J.L. Ávila, M. Mayén, M.L. Mena, J.M. Pingarrón, R. Rodríguez-Amaro, A gold nanoparticle-modified PVC/TTF-TCNQ composite amperometric biosensor for glucose determination, J Electroanal Chem. 634 (2009) 59-63.

[41] H. Ernst, M. Knoll, Electrochemical characterisation of uric acid and ascorbic acid at a platinum electrode, Anal. Chim. Acta. 449 (2001) 129-134.

[42] L.T. DiPasquale, N.G. Poulos, J.R. Hall, A. Minocha, T.A. Bui, M.C. Leopold, Structurefunction relationships affecting the sensing mechanism of monolayer-protected cluster doped xerogel amperometric glucose biosensors, J. Colloid Interface Sci. 450 (2015) 202-212.

[43] S.A. Rothwell, S.J. Killoran, R.D. and O'Neill, Enzyme immobilization strategies and electropolymerization conditions to control sensitivty and selectivity parameters of a polymerenzyme composite glucose biosensor, Sensors. 10 (2010) 6439-6462.

[44] M. Rasia, A. Bollini, Red blood cell shape as a function of medium's ionic strength and $\mathrm{pH}$, Biochimica et Biophysica Acta (BBA) - Biomembranes. 1372 (1998) 198-204.

[45] R.A. Black, D.A. Hill, Over-the-counter medications in pregnancy, Am. Fam. Physician. 67 (2003) 2517-2524.

[46] E. Collins, Maternal and fetal effects of acetaminophen and salicylates in pregnancy, Obstet. Gynecol. 58 (1981) 57S-62S.

[47] M.L. Chang, H. Dong, X. Cao, J.H.T. Luong, X. Zhang, Implantable Electrochemical Sensors for Biomedical and Clinical Applications: Progress, Problems, and Future Possibilities, Curr. Med. Chem. 14 (2007) 937-951.

[48] G.S. Wilson, R. Gifford, Biosensors for real-time in vivo measurements, Biosensors and Bioelectronics. 20 (2005) 2388-2403.

[49] R. Gifford, M.M. Batchelor, Y. Lee, G. Gokulrangan, M.E. Meyerhoff, G.S. Wilson, Mediation of in vivo glucose sensor inflammatory response via nitric oxide release, Biomed Mater Res. 75A (2005) 755-766.

[50] S.M. Muhammet, S. Cete, F. Arslan, A. Yasar, Amperometric cholesterol biosensors based on the electropolymerization of pyrrole and aniline in sulphuric acid for the determination of cholesterol in serum, Artif. Cells Blood Substit. Biotechnol. 37 (2009) 273-278.

[51] L.J. Elsas, K. Lai, The molecular biology of galactosemia, Genet. Med. 1 (1998) 40-48. 
Table 1: Sensor Performance Properties of Xerogel-Based, $1^{\text {st }}$ Generation Amperometric Uric Acid Biosensors

\begin{tabular}{cccccccc}
\hline System & $\begin{array}{c}\text { Sensitivity } \\
(\mathrm{nA} / \mu \mathrm{M})\end{array}$ & $\begin{array}{c}\text { Response } \\
\text { Time } \\
(\mathrm{s})\end{array}$ & $\begin{array}{c}\text { Linear } \\
\text { Range }^{\mathrm{a}}(\mu \mathrm{M})\end{array}$ & $\begin{array}{c}\text { Dynamic }^{\text {Range }} \\
(\mu \mathrm{M})\end{array}$ & $\begin{array}{c}\text { LOD } \\
(\mu \mathrm{M}){ }^{\mathrm{b}}\end{array}$ & $\begin{array}{c}\text { Stability } \\
(\text { days })\end{array}$ & $\begin{array}{c}\text { Shelf- } \\
\text { Life } \\
(\text { days })\end{array}$ \\
\hline Pt/HMTES*/HMTES/PL-A/PU & $0.78_{ \pm 0.11}$ & $10.0_{ \pm 5.3}$ & 700 & 700 & $5.0_{ \pm 2.4}$ & $>10$ & $>30$ \\
Pt/OTMS*/OTMS/ PL-A /PU & $1.02_{ \pm 0.07}$ & $8.0_{ \pm 4.3}$ & 700 & 700 & $2.4_{ \pm 0.9}$ & $>10$ & $>30$ \\
Pt/IBTMS*/IBTMS/ PL-A /PU & $0.79_{ \pm 0.14}$ & $8.0_{ \pm 4.2}$ & 700 & 700 & $3.2_{ \pm 2.6}$ & $>10$ & $>30$ \\
Pt/PTMS*/PTMS/ PL-A /PU & $0.62_{ \pm 0.19}$ & $34.7_{ \pm 12.6}$ & 700 & 700 & $7.9_{ \pm 5.0}$ & N/A $^{\mathrm{c}}$ & N/A $^{\mathrm{c}}$ \\
\hline
\end{tabular}

* indicates UOx doped layer; a. Typical upper limit of range listed; b. IUPAC limit of detection (LOD) is the concentration required to elicit a sensor response $\left(3 \sigma_{\mathrm{BL}} / \beta_{1}\right)$; c. PTMS system was not tested for stability and shelf-life due to having the lowest sensitivity and poorest response time.

Note: A table comparing these performance properties to that of other types of sensors in the literature is available in Supplementary

Materials. 


\section{Captions}

Figure 1. (A) Cyclic voltammetry of $5 \mathrm{mM} \mathrm{FeCN} \mathrm{(0.5} \mathrm{M} \mathrm{KCl,} 100 \mathrm{mV} / \mathrm{sec})$ and (B) amperometric I-t curves during successive injections of $\mathrm{H}_{2} \mathrm{O}_{2}(100 \mu \mathrm{M}$ increases in 4.4 mM PBS, $\mathrm{pH}=7$ ) at (a) unmodified Pt electrode and electrodes modified with a PU coating blends comprised of (b) 100:0, (c) 75:25, (d) 50:50, (e) 25:75 and (f) 0:100 ratios of HPU:TPU; (C) summary comparison of the oxidative peak current for FeCN voltammetry $\left(\square, i_{p, a}\right)$ versus the average amperometric response during $\mathrm{H}_{2} \mathrm{O}_{2}$ injections $\left(\boldsymbol{\Delta}, \mathrm{i}_{\mathrm{a}}\right)$ at various PU coatings; Note: In some cases, error bars are smaller than markers for average.

Figure 2. Average amperometric response (current change) for an injections of UA ( $300 \mu \mathrm{M}$ in 4.4mM PBS, $\mathrm{pH} 4.6)$ at an unmodified Pt electrode (0:0) and electrodes modified with a polyurethane coatings comprised of 0:100, 25:75, 50:50, 75:25 and 100:0 ratios of HPU:TPU.

Figure 3. (A) Calibration curves during successive $1 \mathrm{~mL}$ injections of UA standard $(1.86 \mathrm{mM})$ at a Pt electrode modified OTMS xerogel bilayers capped with PU (100\% HPU) where the first OTMS xerogel layer is doped with different species of uricase enzyme* (UOx) including: Bacillus fastidiosus (BF), BF (Sigma) and Candida sp. (Sigma); (B) Calibration curves during successive $50 \mu \mathrm{M}$ injections of uric acid at a platinum electrode modified with different silane precursor xerogel bilayers, the first layer doped with UOx,* and coated with PU (100\% HPU). Notes: $R^{2}$ values are $\geq 0.999$ in all cases except for APTMS $(\mathbf{x})$ response; Solid symbols indicate a step-like response which was observed in all cases across the concentration range except for APTMS (x). Solution: $65.5 \mathrm{mM}$ PBS, $\mathrm{pH}=7.0 ; \mu=150 \mathrm{mM}$.

Figure 4. (A) Calibration curves during successive $1 \mathrm{~mL}$ injections of UA standard at a $\mathrm{Pt}$ electrode modified with various UOx-doped xerogels, undoped xerogels, as well as selective membranes of electropolymerized PL-A film and PU (100\% HPU); (B) Specific I-t curve example for successive injections of common interferent species and uric acid at the full HMTES xerogel system and; (C) Selectivity coefficient comparisons at the various xerogel systems from (A). Solution: $65.5 \mathrm{mM}$ PBS, $\mathrm{pH}=7.0 ; \mu=150 \mathrm{mM}$; [ $\mathrm{R}^{2} \geq 0.998$ all cases $]$.

Figure 5. (A) Amperometric I- $t$ curve and corresponding calibration curve (inset) during successive $50 \mu \mathrm{M}$ injections of UA at a Pt electrode modified with UOx-doped HMTES xerogel*, undoped HMTES xerogel, PL-A, and PU layer (100\% HPU); (B) amperometric I- $t$ curves and (C) corresponding calibration curves during UA injections at the various stages of LbL construction of the HMTES xerogel-based sensor. Solid symbols indicate a step-like response whereas open symbols indicate a nonstep response (dynamic range). Solution: $65.5 \mathrm{mM} \mathrm{PBS,} \mathrm{pH}$ $=7.0 ; \mu=150 \mathrm{mM}$. Note: In some cases $(\mathrm{C}, \bullet)$, error bars are smaller than markers for average. 
Figure 6. (A) Amperometric I- $t$ curves and (B) selectivity coefficients for successive injections of common interferent species $(40 \mu \mathrm{M})$ and UA $(100 \mu \mathrm{M}$ and/or $300 \mu \mathrm{M})$ at a Pt electrode modified with various stages of LbL construction including (a) UOx-doped HMTES* xerogel, (b) UOxdoped HMTES* xerogel with an additional layer of undoped HMTES xerogel with (c) an additional layer of PL-A and (d) a capping layer of PU (100\% HPU); Solution: $65.5 \mathrm{mM}$ PBS, $\mathrm{pH}=7.0 ; \mu=150 \mathrm{mM}$.

Figure 7. (A) Sensitivity ( $\square$ ) and response time ( $\diamond)$ in addition to (B) selectivity coefficients of common interferents and UA monitored over a ten-day period at HMTES*/HMTES xerogel uric acid biosensors with PL-A and PU layers. Notes: sensors were stored $\left(4-7^{\circ} \mathrm{C}\right)$ immersed in PBS ( $\mathrm{pH} 7 ; 65.55 \mathrm{mM}, \mu=150 \mathrm{mM}$ ); In some cases, error bars are smaller than markers for average (A) or have been omitted for clarity (B) but included in analogous figures in Supplementary Material.

Figure 8. Shelf-life monitoring of (A) Sensitivity ( $\mathbf{(})$ and response time ( $\bullet$ ) in addition to (B) selectivity coefficients of common interferents and UA monitored over a 35-day period at HMTES*/HMTES xerogel uric acid biosensors with PL-A and PU layers. Notes: sensors were stored $\left(4-7^{\circ} \mathrm{C}\right)$ immersed in PBS $(\mathrm{pH} 7 ; 65.55 \mathrm{mM}, \mu=150 \mathrm{mM})$; In some cases, error bars are smaller than markers for average (A) or have been omitted for clarity (B) but included in analogous figures in Supplementary Material. 
Table 1: Sensor Performance Properties of Xerogel-Based, $1^{\text {st }}$ Generation Amperometric Uric Acid Biosensors

\begin{tabular}{|c|c|c|c|c|c|c|c|c|}
\hline System & WE & $\begin{array}{c}\text { Sensitivity } \\
(\mathrm{nA} / \mu \mathrm{M})\end{array}$ & $\begin{array}{c}\text { Response } \\
\text { Time } \\
\text { (s) }\end{array}$ & $\begin{array}{c}\text { Linear } \\
\text { Range }{ }^{\mathrm{a}} \\
(\mu \mathrm{M})\end{array}$ & $\begin{array}{c}\text { Dynamic } \\
\text { Range }^{\mathrm{a}} \\
(\mu \mathrm{M})\end{array}$ & $\begin{array}{l}\text { LOD } \\
(\mu \mathrm{M})^{b}\end{array}$ & $\begin{array}{c}\text { Stability } \\
\text { (days) }\end{array}$ & $\begin{array}{l}\text { Shelf- } \\
\text { Life } \\
\text { (days) }\end{array}$ \\
\hline Pt/HMTES*/HMTES/PL-A/PU & $\mathrm{Pt}$ & $0.78_{ \pm 0.11}$ & $10.0_{ \pm 5.3}$ & 700 & 700 & $5.0_{ \pm 2.4}$ & $>10$ & $>35$ \\
\hline Pt/OTMS*/OTMS/ PL-A /PU & $\mathrm{Pt}$ & $1.02_{ \pm 0.07}$ & $8.0_{ \pm 4.3}$ & 700 & 700 & $2.4_{ \pm 0.9}$ & $>10$ & $>35$ \\
\hline Pt/IBTMS*/IBTMS/ PL-A /PU & $\mathrm{Pt}$ & $0.79_{ \pm 0.14}$ & $8.0_{ \pm 4.2}$ & 700 & 700 & $3.2_{ \pm 2.6}$ & $>10$ & $>35$ \\
\hline Pt/PTMS*/PTMS/ PL-A /PU & $\mathrm{Pt}$ & $0.62_{ \pm 0.19}$ & $34.7_{ \pm 12.6}$ & 700 & 700 & $7.9_{ \pm 5.0}$ & $N / A^{c}$ & $\mathrm{~N} / \mathrm{A}^{\mathrm{c}}$ \\
\hline
\end{tabular}

* indicates UOx doped layer; a. Typical upper limit of range listed; b. IUPAC limit of detection (LOD) is the concentration required to elicit a sensor response

$\left(3 \sigma_{\mathrm{BL}} / \beta_{1}\right)$; c. PTMS system was not tested for stability and shelf-life due to having the lowest sensitivity and poorest response time.

Note: A table comparing these performance properties to that of other types of sensors in the literature is available in Supplementary Materials. 
Graphical Abstract

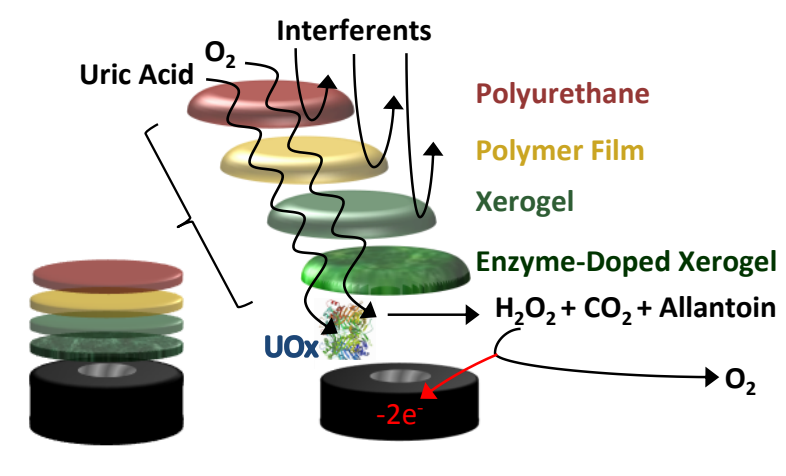

\section{Graphical Abstract}




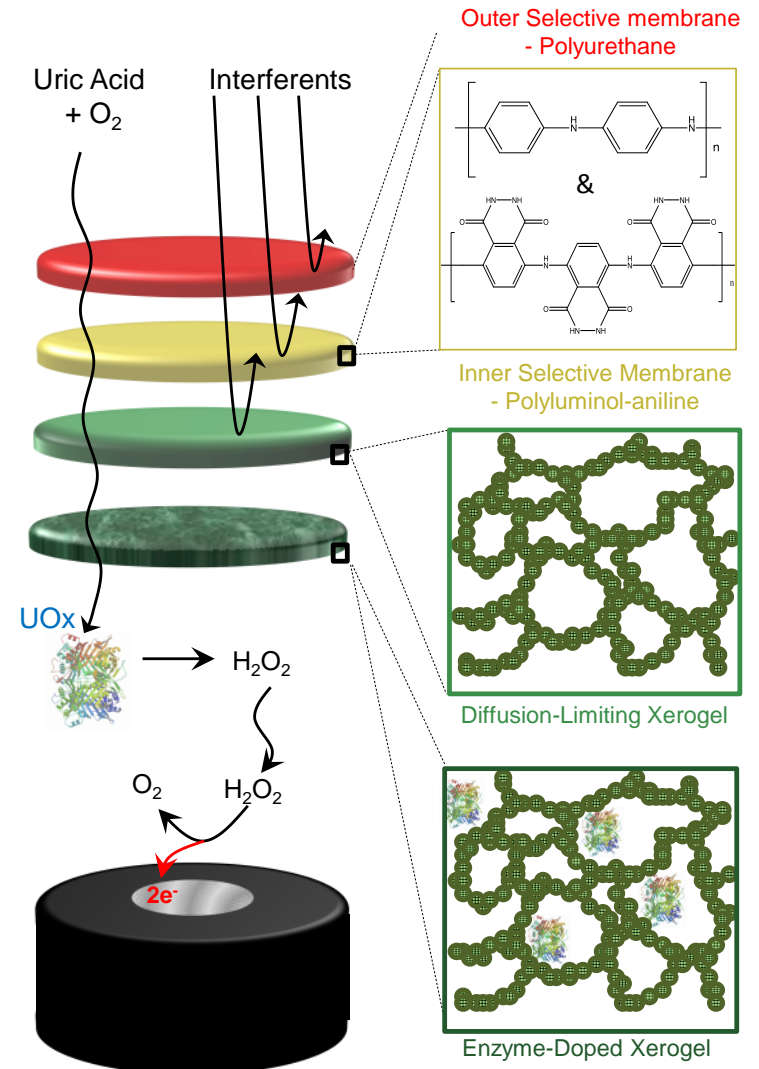



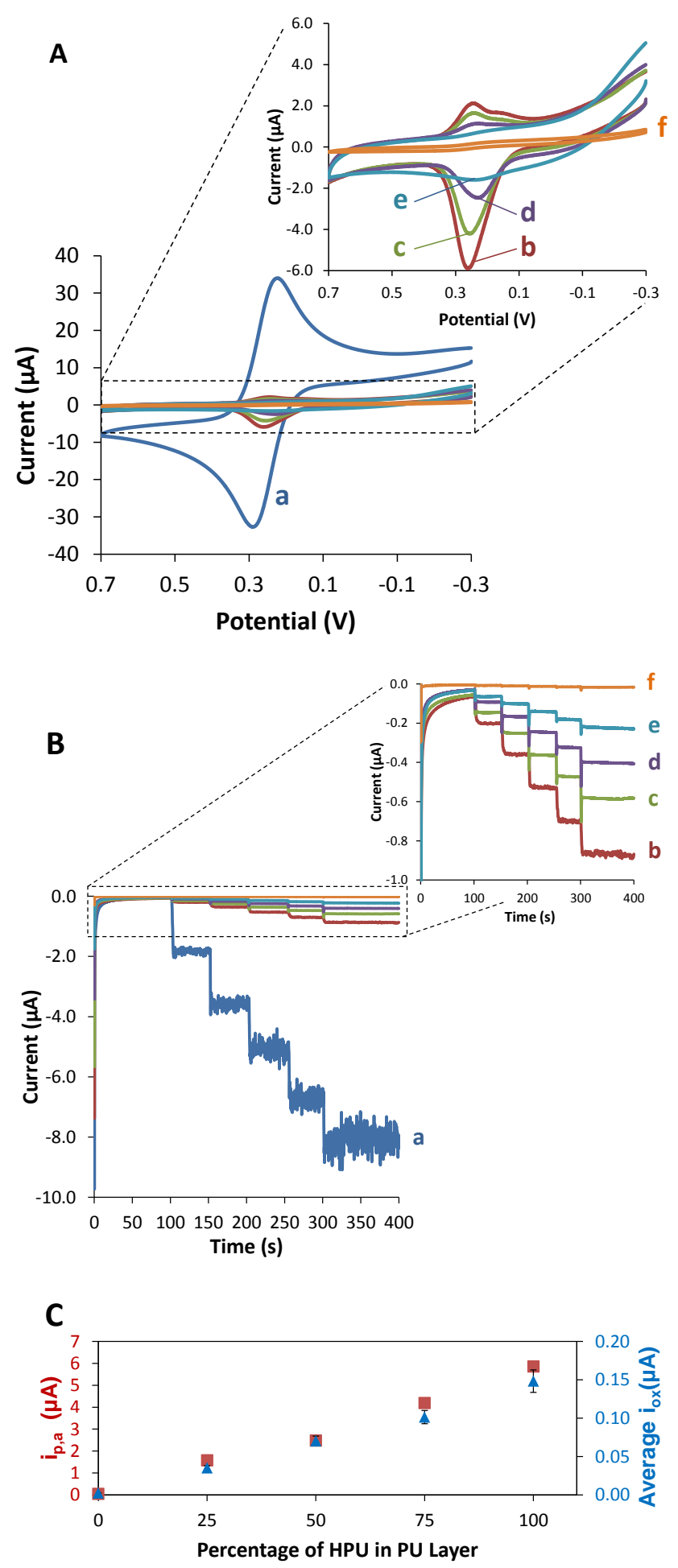

Figure 1. (A) Cyclic voltammetry of $5 \mathrm{mM} \mathrm{FeCN}(0.5 \mathrm{M} \mathrm{KCl}, 100 \mathrm{mV} / \mathrm{sec})$ and (B) amperometric I-t curves during successive injections of $\mathrm{H}_{2} \mathrm{O}_{2}(100 \mu \mathrm{M}$ increases in $4.4 \mathrm{mM} \mathrm{PBS}, \mathrm{pH}=7)$ at (a) unmodified Pt electrode and electrodes modified with a PU coating blends comprised of (b) 100:0, (c) 75:25, (d) 50:50, (e) 25:75 and (f) 0:100 ratios of HPU:TPU; (C) summary comparison of the oxidative peak current for FeCN voltammetry $\left(\boldsymbol{\square}, \mathrm{i}_{\mathrm{p}, \mathrm{a}}\right)$ verses the average amperometric response during $\mathrm{H}_{2} \mathrm{O}_{2}$ injections $\left(\boldsymbol{\Lambda}, \mathrm{i}_{\mathrm{a}}\right)$ at various PU coatings; Note: In some cases, error bars are smaller than markers for average. 


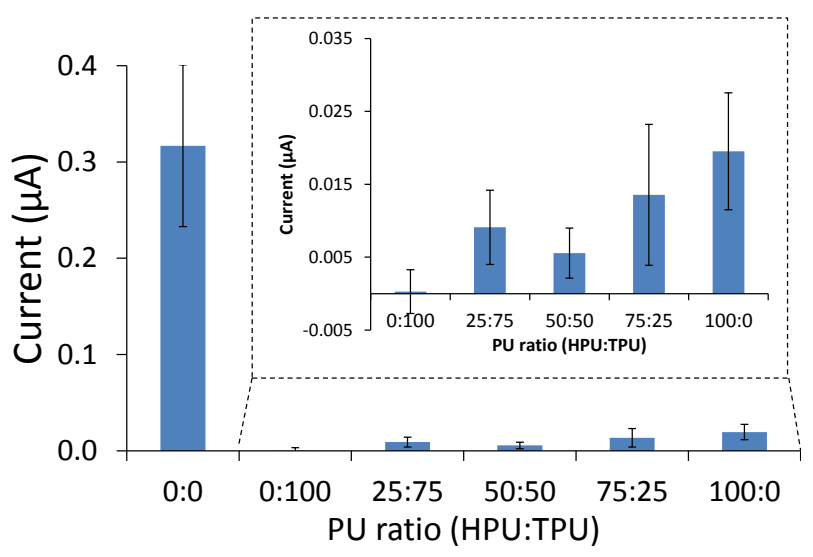

Figure 2. Average amperometric response (current change) for an injections of UA ( $300 \mu \mathrm{M}$ in $4.4 \mathrm{mM}$ PBS, pH 4.6) at an unmodified Pt electrode (0:0) and electrodes modified with a polyurethane coatings comprised of 0:100, 25:75, 50:50, 75:25 and 100:0 ratios of HPU:TPU. 

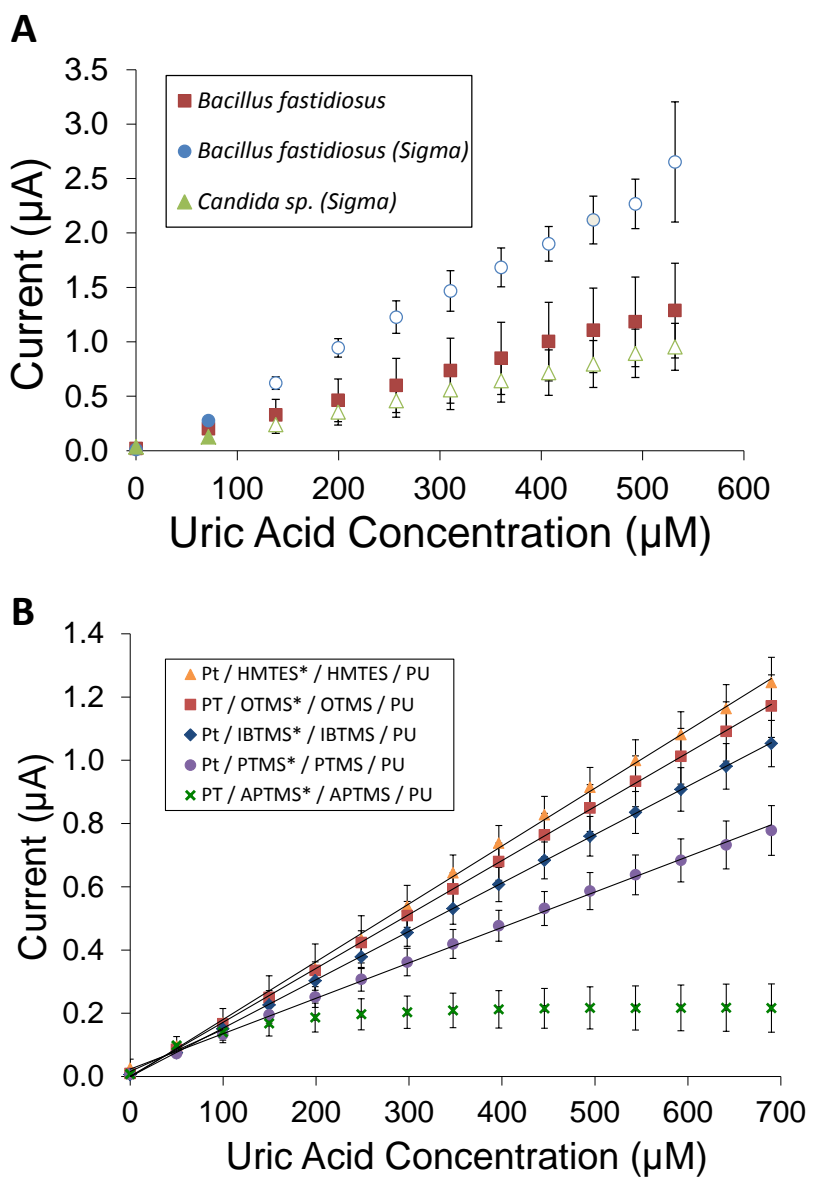

Figure 3. (A) Calibration curves during successive $1 \mathrm{~mL}$ injections of UA standard $(1.86 \mathrm{mM})$ at a $\mathrm{Pt}$ electrode modified OTMS xerogel bilayers capped with PU (100\% HPU) where the first OTMS xerogel layer is doped with different species of uricase enzyme* (UOx) including: Bacillus fastidiosus (BF), BF (Sigma) and Candida sp. (Sigma); (B) Calibration curves during successive $50 \mu \mathrm{M}$ injections of uric acid at a platinum electrode modified with different silane precursor xerogel bilayers, the first layer doped with UOx, ${ }^{*}$ and coated with PU (100\% HPU). Notes: $\mathrm{R}^{2}$ values are $\geq 0.999$ in all cases except for APTMS (x) response; Solid symbols indicate a step-like response which was observed in all cases across the concentration range except for APTMS (x). Solution: $65.5 \mathrm{mM}$ PBS, $\mathrm{pH}=7.0 ; \mu=150 \mathrm{mM}$. 

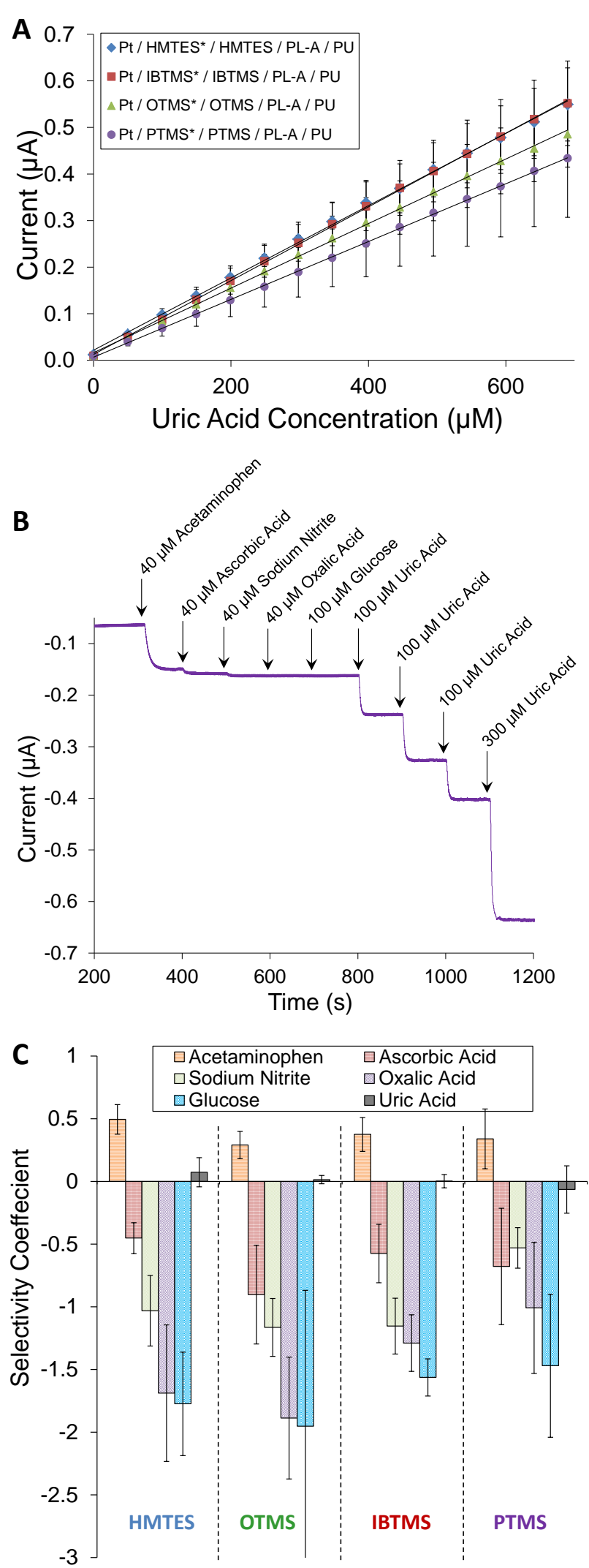

Figure 4. (A) Calibration curves during successive $1 \mathrm{~mL}$ injections of UA standard at a Pt electrode modified with various UOx-doped xerogels, undoped xerogels, as well as selective membranes of electropolymerized PL-A film and PU (100\% HPU); (B) Specific I-t curve example for successive injections of common interferent species and uric acid at the full HMTES xerogel system and; (C) Selectivity coefficient comparisons at the various xerogel systems from (A). Solution: $65.5 \mathrm{mM}$ PBS, $\mathrm{pH}=7.0 ; \mu=150 \mathrm{mM} ;\left[\mathrm{R}^{2} \geq 0.998\right.$ all cases $]$. 

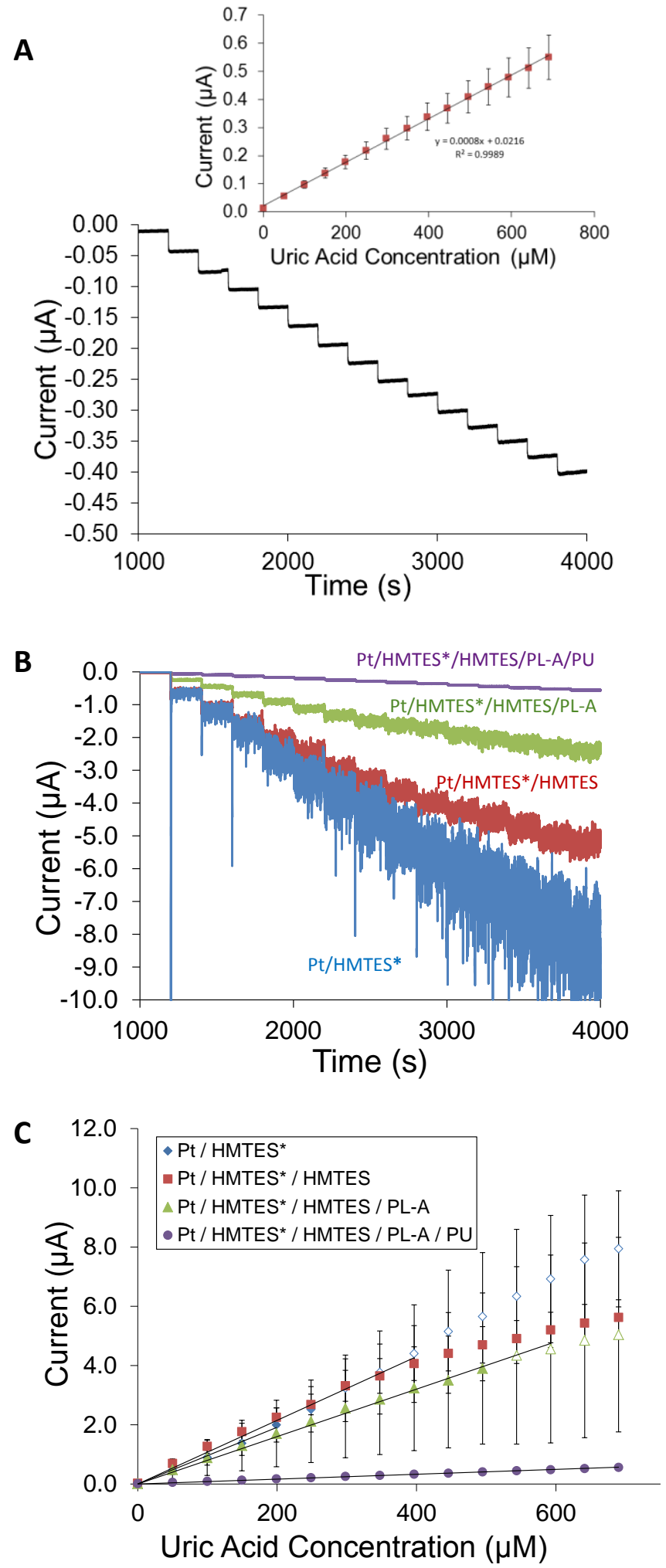

Figure 5. (A) Amperometric I- $t$ curve and corresponding calibration curve (inset) during successive 50 $\mu \mathrm{M}$ injections of UA at a Pt electrode modified with UOx-doped HMTES xerogel*, undoped HMTES xerogel, PL-A, and PU layer (100\% HPU); (B) amperometric I- $t$ curves and (C) corresponding calibration curves during UA injections at the various stages of L-B-L construction of the HMTES xerogel-based sensor. Solid symbols indicate a step-like response whereas open symbols indicate a nonstep response (dynamic range). Solution: $65.5 \mathrm{mM}$ PBS, $\mathrm{pH}=7.0 ; \mu=150 \mathrm{mM}$. Note: In some cases $(\mathrm{C}, \bullet)$, error bars are smaller than markers for average. 

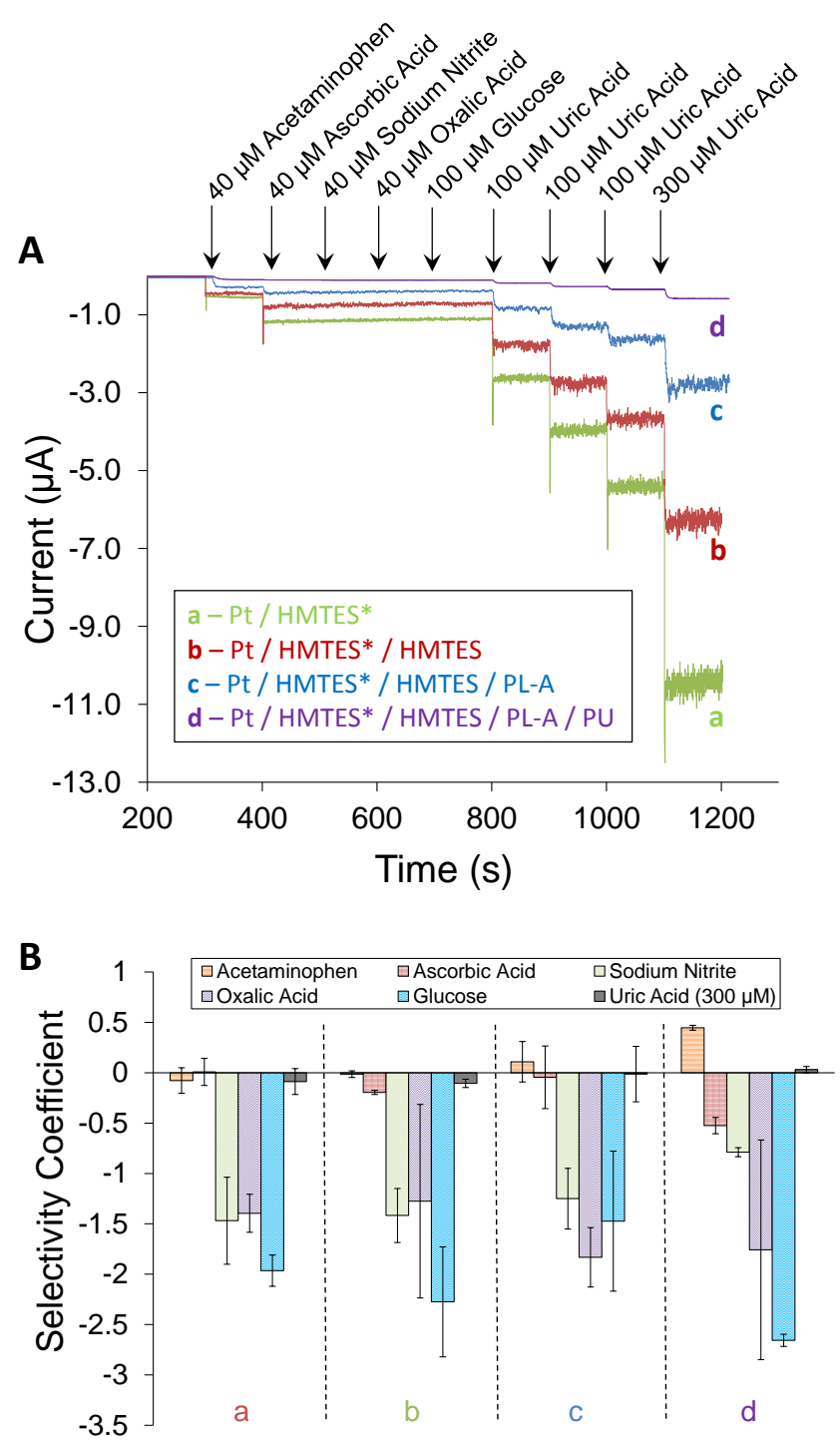

Figure 6. (A) Amperometric I- $t$ curves and (B) selectivity coefficients for successive injections of common interferent species $(40 \mu \mathrm{M})$ and UA $(100 \mu \mathrm{M}$ and/or $300 \mu \mathrm{M})$ at a Pt electrode modified with various stages of L-B-L construction including (a) UOx-doped HMTES* xerogel, (b) UOx-doped HMTES* xerogel with an additional layer of undoped HMTES xerogel with (c) an additional layer of PL-A and (d) a capping layer of PU (100\% HPU); Solution: $65.5 \mathrm{mM} \mathrm{PBS,} \mathrm{pH}=7.0 ; \mu=150 \mathrm{mM}$. 

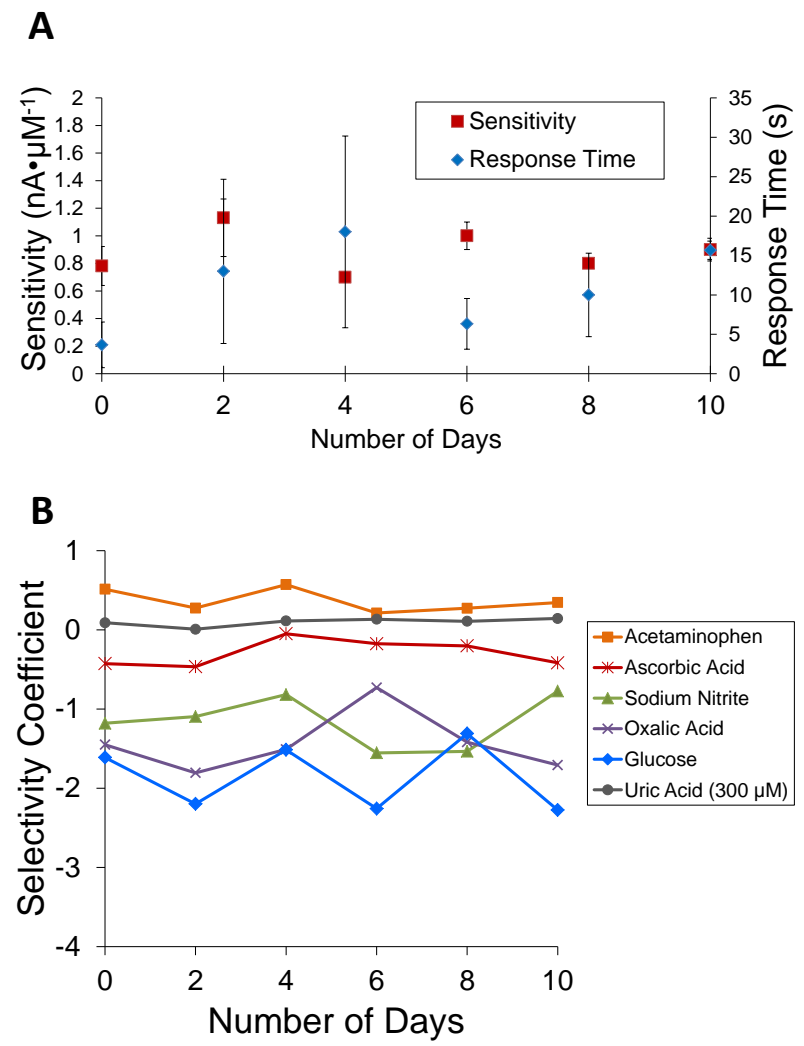

Figure 7. (A) Sensitivity ( $\square$ ) and response time ( $\$$ ) in addition to (B) selectivity coefficients of common interferents and UA monitored over a ten-day period at HMTES*/HMTES xerogel uric acid biosensors with PL-A and PU layers. Notes: sensors were stored $\left(4-7^{\circ} \mathrm{C}\right)$ immersed in PBS $(\mathrm{pH} 7 ; 65.55 \mathrm{mM}, \mu=$ $150 \mathrm{mM}$ ); In some cases, error bars are smaller than markers for average (A) or have been omitted for clarity (B) but included in analogous figures in Supplementary Material. 

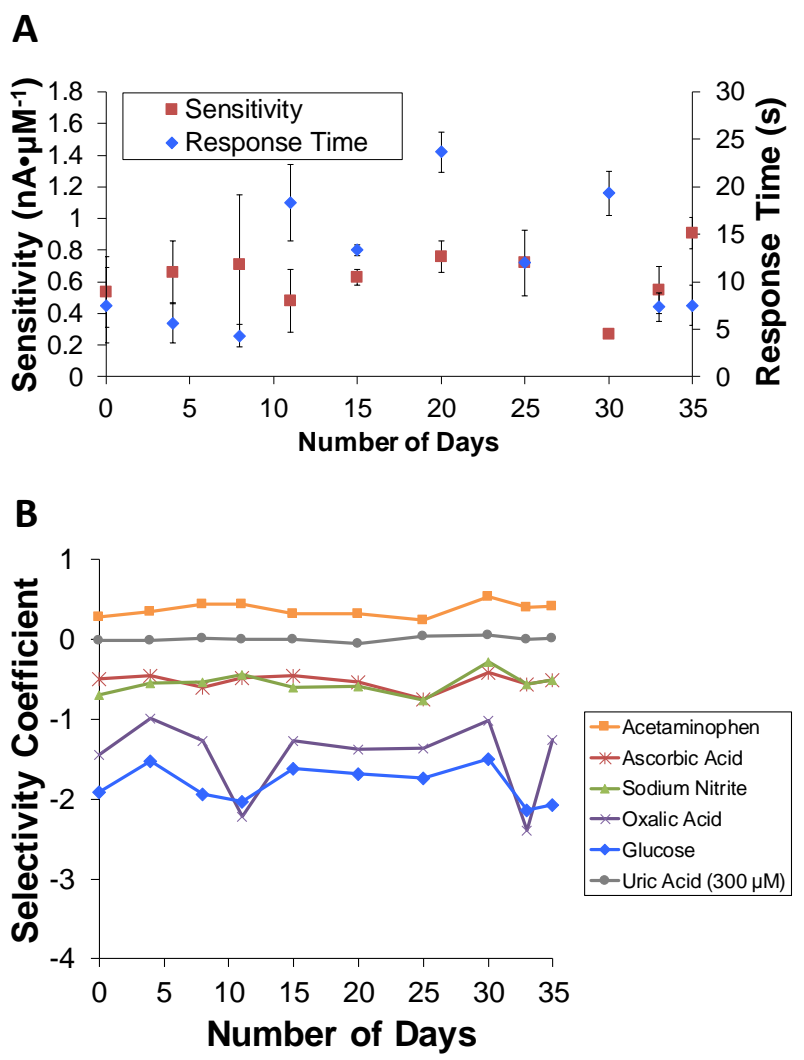

Figure 8. Shelf-life monitoring of (A) Sensitivity ( $\square$ ) and response time ( $\diamond)$ in addition to (B) selectivity coefficients of common interferents and UA monitored over a 30-day period at HMTES*/HMTES xerogel uric acid biosensors with PL-A and PU layers. Notes: sensors were stored $\left(4-7^{\circ} \mathrm{C}\right)$ immersed in PBS (pH 7; $65.55 \mathrm{mM}, \mu=150 \mathrm{mM}$ ); In some cases, error bars are smaller than markers for average (A) or have been omitted for clarity (B) but included in analogous figures in Supplementary Materials. 